\title{
Low dispersion in the infectiousness of COVID-19 cases implies difficulty in control
}

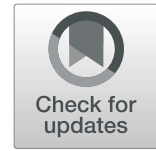

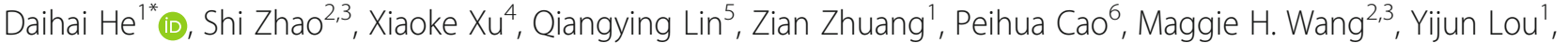 \\ Li Xiao ${ }^{7}$, Ye Wu ${ }^{8,9^{*}}$ and Lin Yang ${ }^{10^{*}}$
}

\begin{abstract}
The individual infectiousness of coronavirus disease 2019 (COVID-19), quantified by the number of secondary cases of a typical index case, is conventionally modelled by a negative-binomial (NB) distribution. Based on patient data of 9120 confirmed cases in China, we calculated the variation of the individual infectiousness, i.e., the dispersion parameter $k$ of the NB distribution, at 0.70 (95\% confidence interval: $0.59,0.98)$. This suggests that the dispersion in the individual infectiousness is probably low, thus COVID-19 infection is relatively easy to sustain in the population and more challenging to control. Instead of focusing on the much fewer super spreading events, we also need to focus on almost every case to effectively reduce transmission.
\end{abstract}

Keywords: COVID-19, Basic reproductive number, Dispersion, Negative binomial, Mitigation

\section{Introduction}

Since the early outbreak of coronavirus disease 2019 (COVID-19) pandemic, huge efforts have been devoted on estimating key epidemiological parameters due to their important implication in mitigation planning. For instance, according to a survey posted in a public domain (https://github.com/midas-network/COVID-19/tree/master/parameter_estimates/2019_novel_coronavirus), there were at least 47 studies (either peer-reviewed or not) on the cumulative case count in a location have been posted, 39 works on the reproductive number $R_{0}$ (number of secondary cases may be cause by a typical primary cases), 13 on the incubation period (time delay between infection and symptom onset), 6 on the serial interval or generation interval (time delay between symptom onset or infection of an index case and its secondary case in a transmission

\footnotetext{
* Correspondence: daihai.he@polyu.edu.hk; wuye@bnu.edu.cn;

l.yang@polyu.edu.hk

${ }^{1}$ Department of Applied Mathematics, Hong Kong Polytechnic University, Hong Kong, China

${ }^{8}$ Computational Communication Research Center, Beijing Normal University, Zhuhai 519087, China

${ }^{10} \mathrm{School}$ of Nursing, Hong Kong Polytechnic University, Hong Kong, China Full list of author information is available at the end of the article
}

chain), 6 on the symptomatic case fatality ratio. However, the individual variation in infectiousness, the dispersion rate $(k)$, has been largely overlooked, except for one early work in Eurosurveillance [1]. He et al. (2020) summarized the recent estimates on $k$ from empirical offspring distributions, including 0.58 (95\% confidence interval [CI]: 0.35, 1.18) of Bi et al. (2020) from a sample of 391 COVID-19 cases in Shenzhen China [2]. It is of note that there is mathematical modelling work based on imported and reported case numbers in a variety of countries showing that $k$ could be 0.1 (95\% CI: $0.05,0.2)$ [3]. The recent study of Lau et al. [4] used a spatiotemporal transmission process model and estimated that overall dispersion parameter $k$ is 0.45 for Cobb County, 0.43 for Dekalb, 0.39 for Fulton, 0.49 for Gwinnett, and 0.32 for Dougherty in Georgia, USA. In this work, with a larger dataset, we calculate $k$ using the empirical offspring distribution approach. Our data are from mainland China where strict surveillance guaranteed the quality of the data. Since we adopted the basic definition approach, our methods do not rely on additional assumptions typically needed for mathematical modelling. 
Table 1 Summary of estimated $R_{0}$ of COVID-19 outbreaks in six countries

\begin{tabular}{lll}
\hline Country & Time interval & $\boldsymbol{R}_{\mathbf{0}}$ \\
\hline France & $20 / 2 / 2020-12 / 3 / 2020$ & $3.5(3.2,3.8)$ \\
& $7 / 2 / 2020-12 / 3 / 2020$ & $2.0(1.7,2.3)$ \\
UK & $21 / 2 / 2020-12 / 3 / 2020$ & $2.9(2.6,3.2)$ \\
& $11 / 2 / 2020-12 / 3 / 2020$ & $2.0(1.7,2.3)$ \\
Singapore & $23 / 1 / 2020-23 / 2 / 2020$ & $1.7(1.4,2.0)$ \\
Germany & $21 / 2 / 2020-12 / 3 / 2020$ & $3.5(3.2,3.8)$ \\
& $11 / 2 / 2020-12 / 3 / 2020$ & $2.3(2.0,2.6)$ \\
Spain & $21 / 2 / 2020-12 / 3 / 2020$ & $3.5(3.2,3.8)$ \\
& $11 / 2 / 2020-12 / 3 / 2020$ & $2.3(2.0,2.6)$ \\
Japan & $23 / 1 / 2020-12 / 3 / 2020$ & $1.7(1.4,2.0)$ \\
& $11 / 2 / 2020-12 / 3 / 2020$ & $2.3(2.0,2.6)$
\end{tabular}

We adopted a similar method as in [1], and simulate a Negative-binomial process to match the observed daily cases in these country over the chosen time period when the number grew exponentially. Using a maximum likelihood approach to infer $R_{0}$. The method is also explained in [6-8]

\section{Method}

Negative binomial distribution (NB) is used to model the distribution of secondary case numbers, i.e., the offspring numbers, of an index case. The dispersion parameter, $k$, (i.e., size, which is nonnegative) controls the variation of the NB distribution. A sufficiently small $k$ implies that the majority of disease transmission was driven by a few superspreaders, and thus the spread is likely to be

Table 2 Frequency of primary cases as a function of the numbers of secondary cases per primary case. Data are from [9]

\begin{tabular}{ll}
\hline Numbers of secondary cases & Frequency of primary cases \\
\hline 0 & 1241 \\
1 & 511 \\
2 & 160 \\
3 & 71 \\
4 & 33 \\
5 & 15 \\
6 & 7 \\
7 & 3 \\
8 & 2 \\
9 & 3 \\
10 & 1 \\
11 & 1 \\
12 & 1 \\
17 & 1 \\
\hline
\end{tabular}

controlled by preventing super-spreading events. A large $k$ implies that the NB distribution approaches a Poisson distribution, and the virus easily persist and is difficult to eradicate. Following the pioneer work of Lloyd-Smith et al. [5], we assume that the number of secondary cases, denoted by $Z$, for a typical primary case, follows $\mathrm{NB}$ (mean $=R_{0}$, dispersion $=k$ ), and thus the variance is $R_{0}+R_{0}^{2} / k$. When $k$ is sufficiently small, the distribution will have a peak at 0 , and in the limit when $k=0$, the NB distribution is concentrated at zero. When $k=1$, the distribution is a geometric distribution; and when $k$ approaches infinity, the NB distribution approaches a Poisson distribution with both mean and variance equal to $R_{0}$ [5].

\section{Results and discussion}

The $k$ plays an important role in explaining the wide spreading of COVID worldwide, given a similar $R_{0}$ as the other coronavirus, i.e., the severe acute respiratory syndrome (SARS). Lloyd-Smith et al. [5] estimated a smaller $k=0.16$ for the SARS outbreak in Singapore in 2003.

We first tried Riou et al.'s [1] method to calculate the $R_{0}$ and $k$ in six countries (see Table 1), and found that $R_{0}$ is in line with World Health Organization (WHO) early estimates, while $k$ cannot be reliably estimated. Then we obtained the numbers of secondary cases from a study by $\mathrm{Xu}$ et al. [9] (see Table 2), and estimated $k=0.7$ (95\%CI $0.59,0.98)$ and $R_{0}=0.69(95 \% \mathrm{CI}: 0.62$, 0.77) using profile likelihood approach and the profile Log likelihood of the NB model given the data in $R_{0}$ versus $k$ plane is shown in Fig 1 . This estimate is larger than that of SARS around 0.16, but close to that of the 1918 pandemic influenza $0.94(95 \%$ CI $0.59,1.72)$ [2]. Our estimate is in line with $\mathrm{Bi}$ et al. 0.58 with 95\% CI: 0.35, 1.18) [10]. However, we have 9120 confirmed cases, compared to $\mathrm{Bi}$ et al. 391 confirmed cases, and thus our estimate has a smaller confidence interval.

Our results suggest that the majority of the COVID-19 transmission is not due to superspreading events. The number of secondary cases of a primary case roughly follows a geometric distribution, large proportion of primary cases have potentials to generate more than one secondary cases. This indicates that COVID-19 is easy to persist in the general population if strong measure is not taken, given the similar $R_{0}$ as SARS. Therefore, outbreak mitigation is relatively difficulty without taking extreme efforts such as city lockdown. 


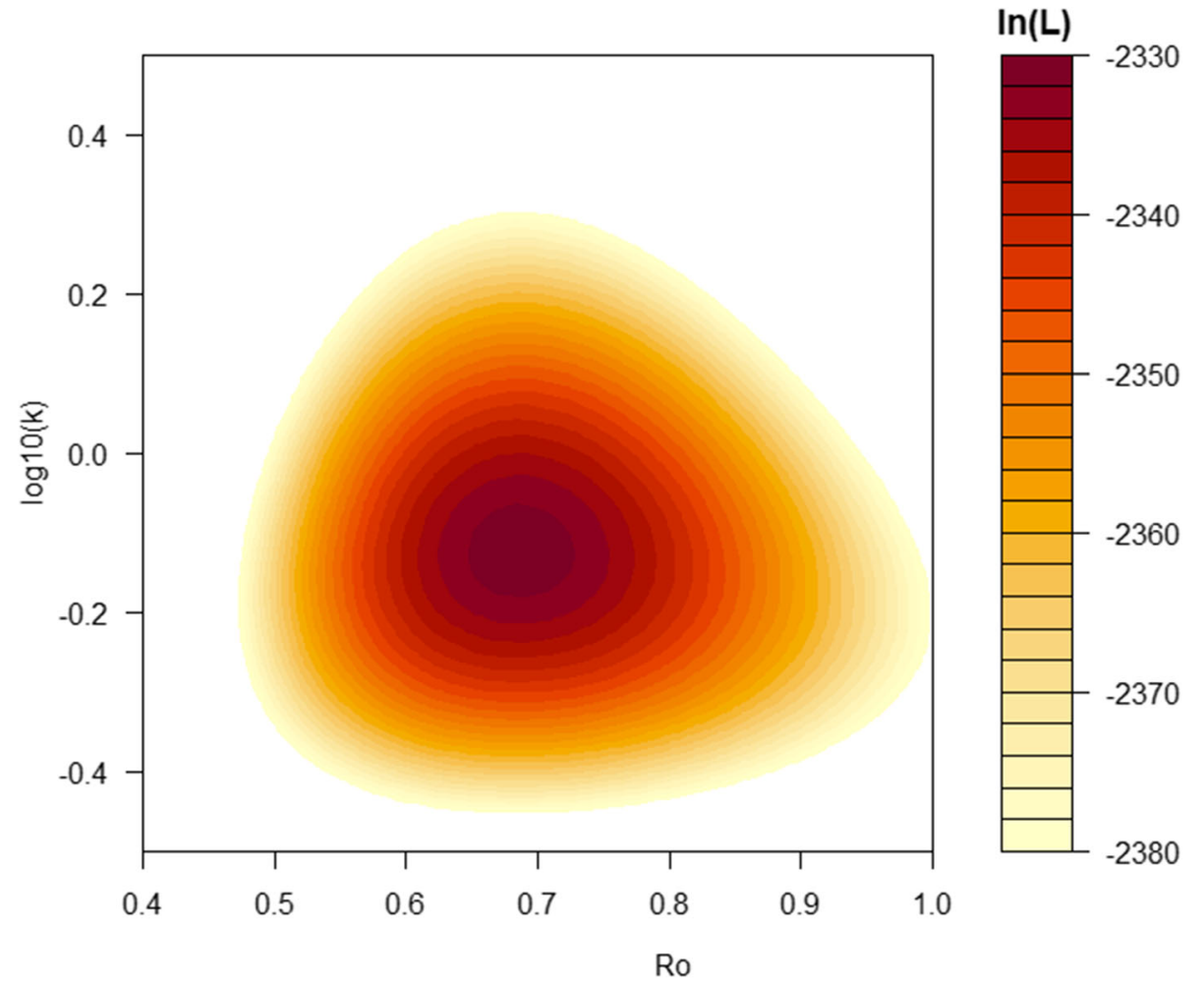

Fig. 1 Profile Log likelihood of the NB model given the data in $R_{0}$ versus $k$ plane based on data listed in Table 2

\section{Abbreviations}

Cl: Confidence interval; COVID-19: Coronavirus disease 2019; NB: Negative binomial distribution; SARS: Severe acute respiratory syndrome

\section{Acknowledgements}

None.

\section{Authors' contributions}

$\mathrm{ZZ}, \mathrm{SZ}, \mathrm{QL}$ and DH conceived the study, carried out the analysis, and drafted the first manuscript. XX, PC, MW, YW, LX, LY and YL discussed the results, critically read and revised the manuscript, and all authors gave final approval for publication.

\section{Funding}

DH was supported by Alibaba (China) Co. Ltd. Collaborative Research project. XX was supported by the National Natural Science Foundation of China (61773091). The funding agencies had no role in the design and conduct of the study; collection, management, analysis, and interpretation of the data; preparation, review, or approval of the manuscript; or decision to submit the manuscript for publication.

\section{Availability of data and materials}

Data used to calculate the $R_{0}$ for six countries are publicly available at https://covid19.who.int/table. Data used to calculate the $k$ for China are given in Table 2

\section{Ethics approval and consent to participate}

The data were collected from public domain, and thus neither ethical approval nor individual consent was not applicable.

\section{Consent for publication}

Not applicable.

\section{Competing interests}

DH was supported by Alibaba (China) Co. Ltd. Collaborative Research project. Other authors declared no conflict of interest.

\section{Author details}

'Department of Applied Mathematics, Hong Kong Polytechnic University, Hong Kong, China. ${ }^{2}$ JC School of Public Health and Primary Care, Chinese University of Hong Kong, Hong Kong, China. ${ }^{3} \mathrm{C} U H K$ Shenzhen Research Institute, Shenzhen, China. ${ }^{4}$ College of Information and Communication Engineering, Dalian Minzu University, Dalian 116600, China. ${ }^{5}$ Michigan Institute for Data Science at University of Michigan, Ann Arbor, MI, USA. ${ }^{6}$ Clinical Research Center, Zhujiang Hospital, Southern Medical University, Guangzhou, China. ${ }^{7}$ College of Medical Information Engineering, Chengdu University of Traditional Chinese Medicine, Chengdu, China. ${ }^{8}$ Computational Communication Research Center, Beijing Normal University, Zhuhai 519087, China. ${ }^{9}$ School of Journalism and Communication, Beijing Normal University, Beijing 100875, China. ${ }^{10}$ School of Nursing, Hong Kong Polytechnic University, Hong Kong, China.

Received: 27 March 2020 Accepted: 29 September 2020

Published online: 16 October 2020

\section{References}

1. Riou J, Althaus CL. Pattern of early human-to-human transmission of Wuhan 2019 novel coronavirus (2019-nCoV), December 2019 to January 2020. Eurosurveillance. 2020;25(4):30

2. Fraser C, Cummings DA, Klinkenberg D, Burke DS, Ferguson NM. Influenza transmission in households during the 1918 pandemic. Am J Epidemiol. 2011;174(5):505-14.

3. Endo A, Abbott S, Kucharski AJ, Funk S. Estimating the overdispersion in COVID-19 transmission using outbreak sizes outside China. Wellcome Open Res. 2020;5(67):67.

4. Lau MS, Grenfell B, Thomas M, Bryan M, Nelson K, Lopman B. Characterizing superspreading events and age-specific infectiousness of SARS-CoV-2 transmission in Georgia, USA. Proc Natl Acad Sci. 2020;117(36):22430-5. 
5. Lloyd-Smith JO, Schreiber SJ, Kopp PE, Getz WM. Superspreading and the effect of individual variation on disease emergence. Nature. 2005;438(7066): 355-9.

6. Althaus CL. Ebola superspreading. Lancet Infect Dis. 2015;15(5):507-8.

7. Zhao S, Cao P, Gao D, Zhuang Z, Chong M, Cai Y, Ran J, Wang K, Lou Y, Wang W, Yang L. Modelling the coronavirus disease (COVID-19) outbreak on the Diamond Princess ship using the public surveillance data from January 20 to February 20, 2020. medRxiv. 2020. https://doi.org/10.1101/2020.02.26. 20028449.

8. Zhuang Z, Zhao S, Lin Q, Cao P, Lou Y, Yang L, Yang S, He D, Xiao L. Preliminary estimates of the reproduction number of the coronavirus disease (COVID-19) outbreak in Republic of Korea and Italy by 5 March 2020 Int J Infect Dis. 2020;95:308-10.

9. Xu XK, Liu XF, Wu Y, Ali ST, Du Z, Bosetti P, Lau EH, Cowling BJ, Wang L. Reconstruction of transmission pairs for novel coronavirus disease 2019 (COVID-19) in mainland China: estimation of super-spreading events, serial interval, and hazard of infection. Clin Infect Dis. 2020;18:ciaa790.

10. Bi Q, Wu Y, Mei S, Ye C, Zou X, Zhang Z, Liu X, Wei L, Truelove SA, Zhang T, Gao W. Epidemiology and transmission of COVID-19 in 391 cases and 1286 of their close contacts in Shenzhen, China: a retrospective cohort study. Lancet Infect Dis. 2020;20(8):911-9.

\section{Publisher's Note}

Springer Nature remains neutral with regard to jurisdictional claims in published maps and institutional affiliations.

Ready to submit your research? Choose BMC and benefit from:

- fast, convenient online submission

- thorough peer review by experienced researchers in your field

- rapid publication on acceptance

- support for research data, including large and complex data types

- gold Open Access which fosters wider collaboration and increased citations

- maximum visibility for your research: over $100 \mathrm{M}$ website views per year

At $\mathrm{BMC}$, research is always in progress.

Learn more biomedcentral.com/submissions 